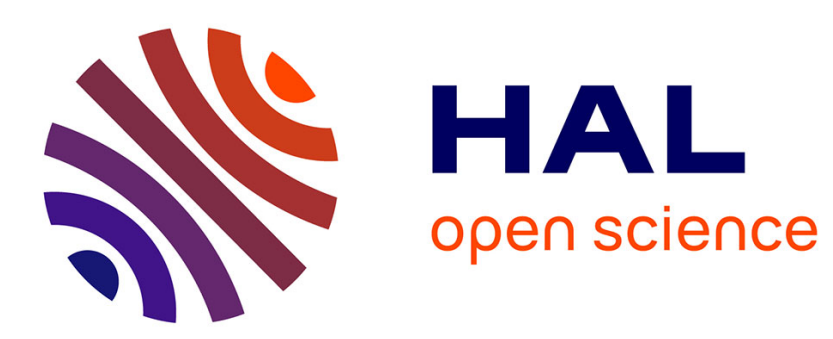

\title{
Study of a thermoelement's behaviour through a modelling based on electrical analogy
}

Gilles Fraisse, Myriam Lazard, Christophe Goupil, Jean-Yves Serrat

\section{To cite this version:}

Gilles Fraisse, Myriam Lazard, Christophe Goupil, Jean-Yves Serrat. Study of a thermoelement's behaviour through a modelling based on electrical analogy. International Journal of Heat and Mass Transfer, 2010, 53 (17-18), pp.3503 - 3512. 10.1016/j.ijheatmasstransfer.2010.04.011 . hal-01923933

\section{HAL Id: hal-01923933 \\ https://hal.univ-smb.fr/hal-01923933}

Submitted on 15 Nov 2018

HAL is a multi-disciplinary open access archive for the deposit and dissemination of scientific research documents, whether they are published or not. The documents may come from teaching and research institutions in France or abroad, or from public or private research centers.
L'archive ouverte pluridisciplinaire HAL, est destinée au dépôt et à la diffusion de documents scientifiques de niveau recherche, publiés ou non, émanant des établissements d'enseignement et de recherche français ou étrangers, des laboratoires publics ou privés. 


\title{
STUDY OF A THERMOELEMENT'S BEHAVIOUR THROUGH A MODELLING BASED ON ELECTRICAL ANALOGY
}

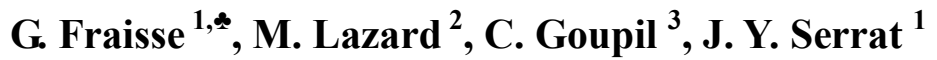 \\ ${ }^{1}$ Laboratoire Optimisation de la Conception et Ingénierie de l'Environnement, \\ LOCIE, Polytech'Savoie, Savoie Technolac, 73376 Le Bourget-Du-Lac, France \\ E-mail: gilles.fraisse@univ-savoie.fr \\ (* corresponding author) \\ 2 Institut Supérieur d'Ingénierie de la Conception, \\ InSIC, 27 rue d'Hellieule, 88100 Saint Dié des Vosges, France \\ Tél : (33) 329421821 - (33) $329422227 \quad$ Fax : (33) 329421825
}

E-mail: mlazard@insic.fr
${ }^{3}$ Laboratoire de Cristallographie et de Science des Matériaux, CRISMAT ENSICAEN, 6 Bd Maréchal Juin 14050 Caen, France
E-mail: christophe.goupil@ensicaen.fr

\section{Abstract :}

This study presents a new thermoelectric element modelling based on an electrical analogy. The balance equation for a node of the analogical model is equivalent to the local energetic balance of a thermoelectric element. A particular attention is paid on taking into account all the effects Peltier, Joule and Thomson. This approach leads to a noticeable modification of the performances compared to previous estimations obtained by classical approaches where the Thomson contribution is neglected and no thermo-dependence of the thermophysical properties is assumed. This analogical modelling is appropriate for description of steady states but also for transient state and for non conventional geometries. The general behaviour of a thermoelectric leg under cooling mode is studied and the different possibilities of improving the performances are investigated. The analysis of the thermodynamical behaviour is also presented by separating the contribution of each effect.

\section{Nomenclature}

$A \quad$ : section of the thermoelectric element $\left(\mathrm{m}^{2}\right)$

COP : coefficient of performance

c : heat capacity $\left(\mathrm{J} \mathrm{kg} \mathrm{K}^{-1}\right)$

$\vec{E} \quad$ : electrical field vector $(\mathrm{V})$ 


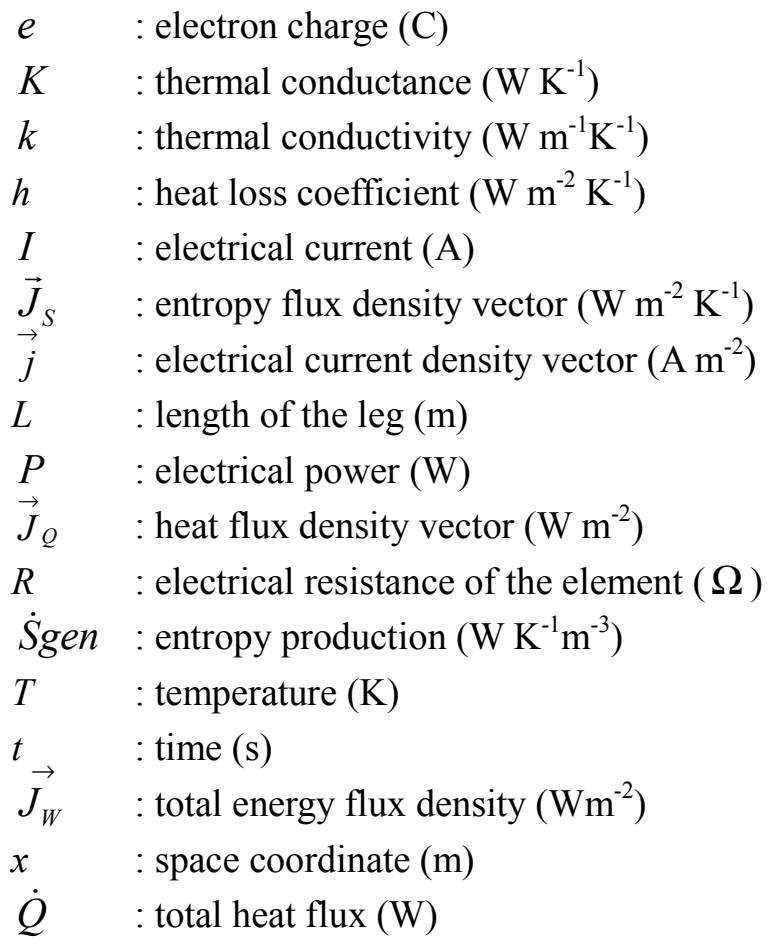

\section{Greek symbols}

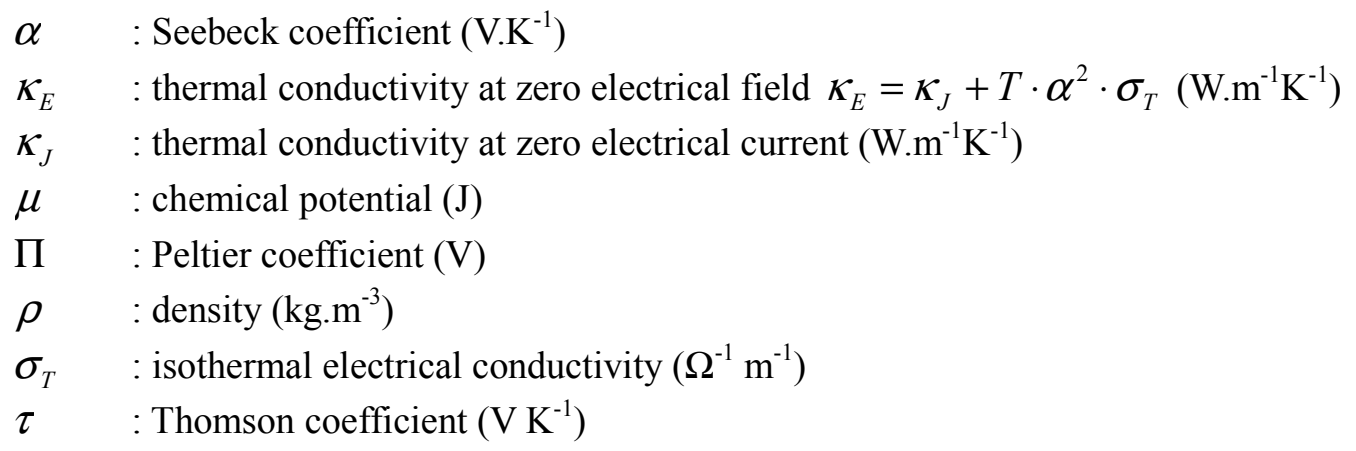

\section{Upperscripts}

C : refers to the cold side $(x=0)$

$\mathrm{H}$ : refers to the hot side $(x=L)$

$\mathrm{N}$ : refers to the N-leg

$\mathrm{P}$ : refers to the P-leg

Keywords :

Modelling, thermoelectric element, heat transfer, temperature-entropy diagram

\section{INTRODUCTION}

Thermoelectric effects have been discovered in the first part of the XIX ${ }^{\text {th }}$ century: Seebeck effect (1821) [1], Peltier effect (1834) [2] and Thomson effect (1851) [3] [4]. Although these physical phenomena are well known, they are not always correctly included in theoretical description and models [5]. For instance, the modeling of the Thomson effect is often neglected 
and, when it is not, some new issues can be observed, as it has been recently reported [6]. The exact contribution of the Thomson effect is also sometimes misleading and it is quite difficult to consider the equations because of the affectation of the sign concerning the Thomson effect. As a consequence there is a doubt on its final contribution on the heating or the cooling of the thermoelectric element.

In this paper, after recalling the basic theoretical background of thermoelectricity deriving from the Onsager's theory $[7,8]$, we develop a method based on an electrical analogy in order to model a thermoelectric element. The Thomson effect and the thermo-dependence of the parameters are all taken into account. Transient states and non conventional geometries are also considered. The results obtained for temperature distribution, fluxes and entropy generation lead to a better understanding of the thermoelement's performances.

\section{MODELLING OF A THERMOELECTRIC ELEMENT}

\subsection{Equations for a unique element $\mathrm{N}$ or $\mathrm{P}$ type.}

The figure 1 shows the principal case-study of this paper. It consists into a single couple of thermoelectric elements of n-type and p-type. The complete hors of equilibrium thermodynamic of the Onsager's development is out of the scope of the present article and only the principal results will be presented, for developed description see references $[7,8,9]$. On the basis of this formalism, the terms of the electrical and thermal fluxes can be written with the following matrix formulation [10] :

$$
\left[\begin{array}{c}
\vec{j} \\
\vec{J}_{Q}
\end{array}\right]=\left[\begin{array}{cc}
\sigma_{T} & \alpha \cdot \sigma_{T} \\
T \cdot \alpha \cdot \sigma_{T} & \kappa_{E}
\end{array}\right] \cdot\left[\begin{array}{c}
\vec{E} \\
-\vec{\nabla} \cdot T
\end{array}\right]
$$

According to the linear response theory, this description only considers the electronic gas. Consequently, the contribution of the lattice to the heat current is neglected in this approach. In order to include the lattice contribution we now consider the total thermal conductivity $\mathrm{k}$ instead of $\kappa_{\mathrm{E}}$.

Eliminating the electrical field $\vec{E}$ in the previous formulation, the heat flux vector within the thermoelectric element (n-type and p-type) is now given by equation (2) [11]:

$\vec{J}_{Q}=-k \cdot \vec{\nabla}(T)+\alpha \cdot \vec{j} \cdot T$

This equation leads to the following expression of the variation of the heat flux $\vec{\nabla} . \vec{J}_{Q}$ :

$\vec{\nabla} \cdot \vec{J}_{Q}=\vec{\nabla} \cdot[\alpha \cdot T \cdot \vec{j}-k \cdot \vec{\nabla}(T)]$

And finally :

$$
\vec{\nabla} \cdot \vec{J}_{Q}=T \cdot \vec{j} \cdot \vec{\nabla}(\alpha)+\vec{j} \cdot \vec{E}-\frac{j^{2}}{\sigma_{T}}-\vec{\nabla} \cdot[k \cdot \vec{\nabla}(T)]
$$

This expression (4) contains four terms which are respectively: 
- the Peltier-Thomson term which account for every variation of the Seebeck coefficient whatever is the variation, isothermal junction or spatial variation due to the effect of the temperature,

- the electrical power supplied,

- the power dissipated by the Joule effect,

- the heat generation due to non isothermal conduction.

The generalized Thomson expression $T \cdot \vec{j} \cdot \vec{\nabla}(\alpha)$ contains both the usual Thomson contribution (effect of the thermal gradient) and the Peltier contribution (effect of the spatial gradient).

Introducing $\Pi=\alpha \cdot T$ and $\tau=d \Pi / d T-\alpha$ it comes :

$T \cdot \vec{j} \cdot \vec{\nabla}(\alpha)=T \cdot \vec{j} \cdot \vec{\nabla}\left(\frac{\Pi}{T}\right)=T \cdot \vec{j} \cdot\left[\frac{1}{T} \vec{\nabla}(\Pi)-\frac{1}{T^{2}} \cdot \Pi \cdot \vec{\nabla}(T)\right]=\vec{j} \cdot[\vec{\nabla}(\Pi)-\alpha \cdot \vec{\nabla}(T)]$

then we get the classical Thomson development [12] writing,

$\vec{\nabla}(\Pi)=\frac{d \Pi}{d T} \cdot \vec{\nabla}(T)$

Then, one obtains:

$T \cdot \vec{j} \cdot \vec{\nabla}(\alpha)=\tau \cdot \vec{j} \cdot \vec{\nabla}(T)$

The expression of the heat flux given by equation (4) is then:

$\vec{\nabla} \cdot \vec{J}_{Q}=\tau \cdot \vec{j} \cdot \vec{\nabla}(T)+\vec{j} \cdot \vec{E}-\frac{j^{2}}{\sigma_{T}}-\vec{\nabla} \cdot[k \cdot \vec{\nabla}(T)]$

Moreover, if the total flux of energy $\vec{J}_{W}$ which includes heat, work and electrochemical flux is considered, it could be written [13]:

$$
\vec{J}_{W}=\vec{q}+\frac{\mu}{e} \cdot \vec{j}
$$

The conservation of energy and of the mass leads to $\vec{\nabla} \cdot \vec{W}=0$ and $\vec{\nabla} \cdot \vec{j}=0$ and then it comes:

$$
\vec{\nabla} \cdot \vec{J}_{Q}=-\vec{\nabla} \cdot\left(\frac{\mu}{e}\right) \cdot \vec{j}=\vec{E} \cdot \vec{j}
$$

The local balance equation can then be considered [14]:

$$
\vec{\nabla} \cdot \vec{J}_{Q}-\vec{E} \cdot \vec{j}=\vec{\nabla} \cdot[k \cdot \vec{\nabla}(T)]+\frac{j^{2}}{\sigma_{T}}-\tau \cdot \vec{j} \cdot \vec{\nabla}(T)=0
$$

All the following quantities are assumed to be constant in the elementary volume cell:

- the section of the element (but can change through the total length)

- the thermal conductivity (which is temperature dependent)

- the current density (which depends on the section)

- the three thermoelectric parameters : Peltier, Seebeck and Thomson coefficients. 
Nevertheless, these values can vary along the thermoelectric leg because the thermophysical parameters (thermoelectric parameters and conductivity) depend on the temperature whereas the current density changes if the section of the elementary volume changes. Assuming one dimensional and steady state conditions, the balance equation of the elementary volume cell becomes:

$\frac{d}{d x}\left(-k \cdot \frac{d T}{d x}+\alpha \cdot j \cdot T\right)=\frac{j^{2}}{\sigma}+\alpha \cdot j \cdot \frac{d T}{d x}$

The parameters $\alpha$ and $\mathrm{j}$ are algebraically defined and their sign depend of the element's nature and of the electrical current orientation (see figure 1).

Under all these assumptions, we get the following equations:

- for a N-leg : $k \cdot \frac{d^{2} T(x)}{d x^{2}}+\frac{j^{2}}{\sigma}-|\tau| \cdot|j| \cdot \frac{d T(x)}{d x}=0$

- for a P-leg: $k \cdot \frac{d^{2} T(x)}{d x^{2}}+\frac{j^{2}}{\sigma}+|\tau| \cdot|j| \cdot \frac{d T(x)}{d x}=0$

In the two legs, the last term is negative because the temperature gradient is positive in the Nleg (and respectively negative in the P-leg). As a consequence, the influence of the Thomson coefficient is the same in the two legs: the Thomson effect contributes to the cooling of the elements between $\mathrm{x}=-\mathrm{L}$ and $\mathrm{x}=+\mathrm{L}$. It is then adequate for thermoelectric modules used for cooling applications. As a general rule, the Thomson effect may lead to a warming up or cooling of the element depending on the sign of the third term [6].

If the transient state is considered, the local balance equation in the N-leg is [13, 14]:

$$
\rho \cdot C p \cdot \frac{d T(x, t)}{d t}=k \cdot \frac{d^{2} T(x, t)}{d x^{2}}+\frac{j^{2}}{\sigma}-|\tau| \cdot|j| \cdot \frac{d T(x, t)}{d x}
$$

The boundary conditions for the thermal problem are imposed temperatures:

$T(x=0, t)=T_{C} \quad$ and $\quad T(x=L, t)=T_{H}$

From the temperature distribution, the total heat flux going through the N-leg is:

$$
\dot{Q}(x, t)=A(x) \cdot\left[|\alpha| \cdot|j| \cdot T(x, t)-k \cdot \frac{d T(x, t)}{d x}\right]
$$

When the section varies for the elementary volume, the balance equation becomes [15]:

$$
C p \cdot \rho \cdot \frac{d T(x, t)}{d t}=\frac{1}{A(x)} \cdot \frac{d}{d x}\left(k \cdot A(x) \cdot \frac{d T(x, t)}{d x}\right)+\frac{j^{2}}{\sigma}-|\tau| \cdot|j| \cdot \frac{d T(x, t)}{d x}
$$

Since the size of the elementary cell is small we can neglect the contribution of the term $\frac{1}{A(x)} \cdot \frac{d A(x)}{d x} \cdot \frac{d T(x, t)}{d x}$. Then using a general analogical model approach, we replicate the thermoelectric elementary cell along the complete leg. The same method is used to take into account the effect of the temperature on the different thermoelectric parameters $(\alpha, \tau, \Pi)$ and on the thermophysical parameters $(k, C p, \rho)$. Even if these parameters are supposed to be constant in each cell, their variation along the whole leg can be correctly taken into account. 
It should be noticed here that the Thomson effect is often neglected in such descriptions [15, 16]. We show here that this is an error since its role is not negligible at all $[17,18]$. It is even possible to improve the performances of a thermoelectric element using cross-contributions of the merit figure $\mathrm{Z}$ and the Thomson effect [6].

\subsection{Analogical modeling of a thermoelectric element}

The electrical analogy allows to easily describe complex phenomena such as the thermal dependence of the thermoelectric parameters, the transient state, the variable section of the thermoelement and other linear processes. The electrical analogy is schematically represented in the figure 2 for cells with a thickness equal to $\Delta x$.

The thermophysical parameters are temperature dependent and the section $A(x)$ can also vary. As a consequence, the parameters of the analogical model, i.e. the thermal capacitances and resistances, are given by the following expressions:

$$
\begin{aligned}
& C_{n}=\rho(T) \cdot C p(T) \cdot A(x) \cdot \Delta x \\
& R_{n}=\frac{\Delta x}{k(T) \cdot A(x)}
\end{aligned}
$$

with $n$ the number of the considered node.

The Joule and Thomson effects are taken into account through the heat flux $\Phi_{n}$ injected in each node of the temperature. For a N-leg the expression is given by:

$$
\Phi_{n}=R_{e} \cdot I^{2}-|\tau| \cdot|I| \cdot \frac{T_{n+1}-T_{n-1}}{2}
$$

where $R_{e}=\frac{\rho_{e}(T) \cdot \Delta x}{A(x)}$ is the electrical resistance of slice whose thickness is equal to $\Delta x$.

It is then possible to determine the total heat flux $\dot{Q}_{n}$ within the thermoelement:

$$
\dot{Q}_{n}=|\alpha| \cdot I \cdot T_{n}-k(T) \cdot A(x) \cdot \frac{T_{n+1}-T_{n-1}}{2 \cdot \Delta x}
$$

\subsection{Other modeling approaches}

A very simple model for a thermoelement can be directly derived from the energy global balance, assuming that the joule production is equally shared at the hot and the cold side. For large section or material, the validity of this is questionable but in the present, since each cell is consider to be isothermal, this assumption becomes totally valid. We consider here a thermoelectric leg with a positive Seebeck coefficient and an electrical current flowing along the increasing $\mathrm{x}$ direction.

At the cold side $(x=0)$ and at the hot side $(x=L)$, the expressions of the heat fluxes are then,

$$
\begin{aligned}
& \dot{Q}_{C}=\alpha \cdot I \cdot T_{C}-K \cdot \Delta T-\frac{1}{2} \cdot R \cdot I^{2}+\frac{1}{2} \cdot \tau \cdot I \cdot \Delta T \\
& \dot{Q}_{H}=\alpha \cdot I \cdot T_{H}-K \cdot \Delta T+\frac{1}{2} \cdot R \cdot I^{2}-\frac{1}{2} \cdot \tau \cdot I \cdot \Delta T
\end{aligned}
$$


where $\mathrm{K}$ is the thermal conductance between the two faces, $\mathrm{R}$ the electrical resistance of the element and $\Delta T=T_{H}-T_{C}$.

In the previous expressions of the heat fluxes, the Thomson effect has been taken into account, which is rarely the case for such studies [16, 19, 20, 21, 22, 23].

If the parameters and the section are constants all over the leg then it is possible to determine the analytical solution for steady state cases, taking into account the contribution of the Thomson effect [14]. Heat flux and the temperature solutions are then:

$$
\begin{aligned}
& \dot{Q}(x)=\alpha \cdot I \cdot T(x)-\frac{k \cdot I}{\tau \cdot \sigma}+\frac{\left(-Q_{F}+\frac{k \cdot \rho_{e} \cdot I}{\tau}\right) \cdot \frac{Q_{T}}{Q_{F}}}{\exp \left[\frac{Q_{T}}{Q_{F}}\left(1-\frac{x}{L}\right)\right]-\exp \left[-\frac{Q_{T}}{Q_{F}} \frac{x}{L}\right]} \\
& T(x)=T_{C}+\left(T_{H}-T_{C}-\frac{c}{b} L\right) \cdot \frac{\exp (-b \cdot x)-1}{\exp (-b \cdot L)-1}+\frac{c}{b} x \\
& \text { where } b=-\frac{\tau j}{k} \quad c=-\frac{j^{2}}{k \cdot \sigma} \quad Q_{F}=k \cdot A \cdot \frac{\Delta T}{L} \quad Q_{T}=\tau \cdot I \cdot \Delta T
\end{aligned}
$$

The electrical output power is:

$$
P=\dot{Q}_{H}-\dot{Q}_{C}
$$

The analytical and simplified solutions give:

$P=R \cdot I^{2}+(\alpha-\tau) \cdot I \cdot \Delta T \quad$ (the parameters are assumed to be positive)

The temperature dependence of the coefficients is usually not considered in the literature, and only averages values between hot and cold side are taken into account [12]. But the temperature dependence is far from being a small effect when considering the Temperature-Entropy diagram [14].

\subsection{Evaluation of the performance}

In order to qualify the performances of a thermoelectric module for cooling applications, we need to define two terms:

- the performance coefficient

$$
\begin{aligned}
& C O P=\frac{\dot{Q}_{C}}{\dot{Q}_{H}-\dot{Q}_{C}} \\
& \dot{Q}_{C}
\end{aligned}
$$

When dealing with performance optimization, it is usually not obvious to find a compromise between the values of COP and $\dot{Q}_{C}$. This can be achieved in modelling by varying the parameters values. For instance, the length of the leg is optimal when the thermal gradient is equal to zero for $x=L$ because $\dot{Q}_{H}$ is then maximum [24]. The introduction of the effect of the Thomson coefficient on the performances can be found in the reference [17], and the optimization of the geometry is achieved in the references $[19,24]$. Using transient mode, the over-cooling has also been studied thanks to pulsed intensity and duration [12, 16, 23]. 
From a thermodynamical point of view, the behaviour of a thermoelectric element can be described using a flux-force approach [12, 14, 20, 24].This thermodynamical analysis give insight into the root causes of the entropy production. Then, an optimization of the working cycle can be considered using the Temperature-Entropy diagram (T-S). The two main root causes are well known since they are due to the internal Joule effect and, in a less contribution, to the non isothermal heat conduction. In the T-S diagram the COP is directly given by the surfaces of the diagram, which are currently symmetrical, and consider constant parameters.

According to the previous expressions of the fluxes, we can easily get the expression of the entropy flux density $\overrightarrow{J_{S}}[24]$ :

$\overrightarrow{J_{S}}=\frac{-k \cdot \vec{\nabla}(T)}{T}+\alpha \cdot \vec{j}$

In the N-leg, this expression becomes:

$J_{S}(x)=\frac{-k}{T(x)} \cdot \frac{d T(x)}{d x}+|\alpha| \cdot|j|=\frac{\dot{Q}(x)}{A(x) \cdot T(x)}$

Assuming constant coefficients and purely adiabatic conditions, the analytical solution $J_{S}(x)$ can easily be determined from the expression $\dot{Q}(x)$ [14].

For the steady state case and for adiabatic conditions, the local creation of entropy is:

$$
\dot{S} g e n=\frac{j^{2}}{\sigma \cdot T}-k \cdot \nabla(T) \cdot \nabla\left(\frac{1}{T}\right)
$$

For the whole system, the total production of entropy is then:

$$
\dot{S} g e n=\frac{\dot{Q}_{H}}{T_{H}}-\frac{\dot{Q}_{C}}{T_{C}}=\left(J_{S}\right)_{H} \cdot A_{H}-\left(J_{S}\right)_{C} \cdot A_{C}
$$

with $\quad x=0, A(x)=A_{C}$ and $J_{S}(x)=\left(J_{S}\right)_{C}$

$x=L, \quad A(x)=A_{H} \quad$ and $\quad J_{S}(x)=\left(J_{S}\right)_{H}$

From the analytical expression of $\mathrm{Q}(\mathrm{x})$ (in the steady-state case and constants coefficients), we can now derive total entropy production as follow :

$$
\dot{S} g e n=\frac{K \cdot R \cdot I}{\tau} \cdot\left(\frac{1}{T_{C}}-\frac{1}{T_{H}}\right)+\frac{\tau \cdot I \cdot \Delta T-R \cdot I^{2}}{1-\exp (\tau \cdot I / K)} \cdot\left[-\frac{1}{T_{C}}+\frac{\exp (\tau \cdot I / K)}{T_{H}}\right]
$$

The Thomson effect is reversible and its contribution to the total entropy production is directly due to the temperature gradient within the thermoelectric leg. Taking the Thomson effect into account in the expressions of $\dot{Q}_{C}$ and $\dot{Q}_{H}$, we get :

$$
\dot{S} g e n=\frac{1}{2} \cdot\left(R \cdot I^{2}-\tau \cdot I \cdot \Delta T\right) \cdot\left(\frac{1}{T_{H}}+\frac{1}{T_{C}}\right)-K \cdot \Delta T \cdot\left(\frac{1}{T_{H}}-\frac{1}{T_{C}}\right)
$$

Next, the local production of entropy for transient state and temperature dependent coefficients can also be evaluated thanks to the analogical scheme,

$$
\dot{S} \operatorname{sen}_{n}=\frac{\left(J_{S}\right)_{n+1} \cdot A_{n+1}-\left(J_{S}\right)_{n-1} \cdot A_{n-1}}{2 \cdot \Delta x \cdot A_{n}}
$$


The optimal configuration obtained by the minimization of the produced entropy [20]. The equivalence between the maximization of the electrical production and the minimization of the produced entropy is presented in [25]. But it is usually preferred to maximize the electrical production because the entropy minimization needs precautions [20]. Moreover, the maximization of the electrical production and the minimization of the production of entropy are not equivalent since the maximal operating point for electrical production can lead to large production of entropy due to non optimized heat transfer. When considering cooling applications, the generated entropy should be compared with the optimization of the COP and $\dot{Q}_{C}$.

\section{STUDY OF THE ELEMENT'S BEHAVIOUR FOR A COOLING USE: POSSIBLE WAYS TO PERFORMANCE IMPROVEMENT}

In this section, all the thermoelectric parameters will be assumed to be positive. Using the analogical approach we take into account the thermal dependence of the parameters for a N-leg.

\subsection{Thomson effect contribution}

The result of the evaluation of the COP and $\dot{Q}_{C}$ either with the analytical or simplified model are plotted in the figure 3 as functions of the electrical current intensity. The parameters values of the model are summarized in table 1 . One can notice that the introduction of the Thomson coefficient contribution modifies the COP value from $18 \%$, if compared to the analogical solution which takes into account the temperature dependence and consequently the Thomson contribution. We see that the inclusion of the Thomson coefficient into the simplified model can be sufficient to obtain a very good estimation of the COP value, which appears to be close to those obtained with the analytical solution with constants parameters. We see that the introduction of the temperature dependence of the parameters slightly reduces the maximal value by $3.5 \%$.

We also confirm that the best cooling performances are obtained when the Thomson coefficient exhibits large values. This phenomenon is observed for both the COP and the cold production $\dot{Q}_{C}$ as it is shown on the figure 4 for current intensities from 1A to 9 A. An increase of $200 \%$ of the Thomson coefficient $\tau$ leads to an improvement of the COP and of $\dot{Q}_{C}(52 \%$ for the COP and $32 \%$ for $\dot{Q}_{C}$ ).

\subsection{Temperature influence}

Up to now we have considered analogical and analytical solutions compared to the constants coefficients case. We see that the results obtained for the temperature field $T(x)$ and the heat flux $Q(x)$ with these two models are the same. On the contrary, when the coefficients are temperature dependent, which is only possible for the analogical model, then the heat flux 
repartition is slightly changed (figure 5) reducing the COP by a small amount. More precisely,

$\dot{Q}_{C}$ gets smaller whereas the difference $\dot{Q}_{H}-\dot{Q}_{C}$ becomes larger with temperature dependence of the coefficients. As far as the temperature distribution is concerned, the differences are very small. These results have been obtained for a small temperature difference between the cold and the hot side. But obviously, a larger temperature difference between the cold and the hot sides will lead to larger difference between the analogical and analytical results.

In the figure 6, the evolutions of the parameters are plotted as functions of the temperature. Since the temperature difference is small $\left(\Delta T=30^{\circ} \mathrm{C}\right)$, the observed variations are almost linear, and the larger difference compared to the mean value is $8 \%$ for the electrical resistivity.

\subsection{Complete contribution}

The figures 7 and 8 show the contribution of all the previous effects on the distribution of the heat flux and the temperature. When only considering the conduction contribution (case 1), we observe an obvious large heat flux. Next we introduce the Peltier contribution (case 2) respectively by adding $\alpha \cdot I \cdot T_{C}$ on $Q_{C}$ and $\alpha \cdot I \cdot T_{H}$ on $Q_{H}$. Then Joule contribution (case 3) is added, $Q_{C}$ and $Q_{H}$ are respectively reduced and increased by $0.5 \cdot R \cdot I^{2}=0.0205 \mathrm{~W}$, using the assumption of symmetrical repartition of the Joule contribution. This lead to lowering the COP. The influence of the Thomson effect is inverse to the contribution of the Joule effect since it tends to cool the thermoelectric element. The Thomson contribution has a symmetrical repartition between the two sides and the variation is about $0.5 \cdot \tau \cdot I \cdot \Delta T=0.00312 \mathrm{~W}$.

The figure 9 shows the contribution of each previous term in the T-S diagram. The total production of entropy in the whole element is proportional to the length of the legs since the section is assumed to be constant. No entropy production can be evidenced by the Peltier effect (1) since the total Peltier heat flux average to zero. The entropy produced by pure thermal conduction (2) is lower than those produced by the Joule effect (3). When introducing the Thomson contribution, the entropy production is slightly reduced. Introducing the temperature dependence of the parameters leads to an increase of the entropy production (4 and 5).

The figure 10 shows the cycling transformations in the T-S diagram, following the current going through the N-leg:

- 1-2: isothermal transformation

- 2-3: adiabatic and irreversible transformation

-3-3: isothermal transformation

The real COP $C O P_{\text {real }}=\frac{\dot{Q}_{C}}{\dot{Q}_{H}-\dot{Q}_{C}}$ is obtained by the ratio of the area delimited by 3-4-10-9 over the area delimited by 1-2-8-9-3-4. 
The Carnot COP COP Carnot $=\frac{T_{C}}{T_{H}-T_{C}}$ is obtained by the ratio of the area delimited by 4-6-710 over the area delimited by 1-5-7-10.

\subsection{Variable section influence}

The figure 11 shows the evolution of the COP, of $\dot{Q}_{C}$, and of $S_{\text {gen }}$ for various conical geometries of the leg. The section at the hot side is $\mathrm{A}_{\mathrm{H}}$ and $\mathrm{A}=1.410^{-6} \mathrm{~m}^{2}$ (constant section as indicated in table 1). The volume and the length of the conical leg are constant $\left(1.9610^{-9} \mathrm{~m}^{3}\right.$ and $\mathrm{L}=1.410^{-3} \mathrm{~m}$ ). When the ratio $\mathrm{A}_{\mathrm{H}} / \mathrm{A}$ is equal to 1 , the section is constant. The model is based on the electrical analogy with thermodependent thermoelectric coefficients. For a constant section leg, the COP reaches a maximum of 0.89 at $2 \mathrm{~A}$. This geometrical configuration leads to a minimum of the entropy production. If we now consider an increase of the section at the hot side (i.e. a reduction of the section at the cold side), the COP decreases to 0.72 but the heat flux $Q_{C}$ slightly increases. Nevertheless, a too small section at the ends of the leg will limit the exchange between the thermoelement and the exchangers leading to poor performances of the overall system.

As expected the entropy production is maximal when the section is minimal, it is due to the dissipation caused by the Joule effect. This is the major contribution to the reduction of the performances, so we now consider a possible extraction of the entropy by a thermal connection of the middle of the leg with a third thermal source, acting as a trash to evacuate a fraction of the Joule production. This cooling can be obtained for instance by blowing air on the element or using micro-caloducs. The total efficiency of the system including this third thermal source will of course decrease, but the local COP of the element will be enhanced. In the figure 12, the COP is plotted as a function of the percentage of the total Joule effect extracted from the leg for a current equal to $2 \mathrm{~A}$. This value of the $\mathrm{COP}$ is compared to the value of the $\mathrm{COP}_{\text {Carnot }}$. Due to thermal conduction entropy, the COP never reaches the Carnot value.

\subsection{Over-cooling and pulsed current}

The effect of a pulsed current has been studied by several authors $[12,16,23]$ since this correspond to a sudden strong increase of the Peltier heat pumping. The cooling is rapidly obtained on the cold side when the pulsed current is applied because the Peltier effect acts instantaneously, while the Joule heat diffuses slowly due to thermal inertia. It is then possible to obtain an over-cooling compared to the steady-state case. Unfortunately, the Joule heat finally diffuses and a large over-heating occurs. The global efficiency of the operation is clearly not satisfactory. We present in the figure 13 the temperature $\mathrm{T} 1$ measured very close to the cold side (boundary condition corresponding to heat flux equal to zero) and T20 close to the hot side (boundary condition corresponding to imposed temperature equal to $308 \mathrm{~K}$ ). The steady state current is $0.96 \mathrm{~A}$ and the pulsed value is $2.1 \mathrm{~A}$, during 2 seconds ( $\mathrm{t}=100 \mathrm{~s}$ to $\mathrm{t}=102 \mathrm{~s})$. 
The figure 14 underlines the very local and brief nature of the cooling effect. One can see that it only occurs for $10 \%$ of the total length of the leg and for a very short duration. According to this observation the current pulse operation is advantageous only for short time over-cooling. The exact duration of the over cooling is given by the thermal inertia of the thermoelectric leg which is here close to 6 seconds [15]. Such a device has been proposed in [14] with high intensity operating point. In order to avoid any diffusion of heat into the cold zone (cold source), it is proposed to thermally disconnect the thermoelement and the cold zone just after the overcooling in order to let the extra Joule effect diffuse into the hot source. This seems to be unlikely since the well known advantage of the Peltier module is this absence of any moving part, the thermal disconnection process would reduce to nothing this advantage. On the other hand the use of a third thermal source, previously proposed, could be a solution for the extraction of this extra Joule heat, leading to a possible increase of the maximal $\Delta \mathrm{T}$ between hot and cold source, actually close to $70 \mathrm{~K}$ for the best Peltier refrigerating systems.

\section{CONCLUSION}

We have presented an electrical analogy model of thermoelectric element. It is shown that this model is able to take into account complex phenomena and various experimental conditions. This compact model can be easily inserted in a thermoelectric device or directly coupled with heat exchangers. Lateral heat losses (convective and radiative exchanges) can easily be added to the elementary cell in order to treat more complex configurations. The behaviour of a thermoelectric element has been compared using various models and some ways to improve the performances have been proposed (influence of the Thomson coefficient, of the geometry, of the current pulse). The role of each phenomenon has been investigated and evaluated by considering the temperature and the heat fluxes within the thermoelectric element. The results obtained have proved the assumption of symmetrical repartition of the Joule effect for the evaluation of $Q_{H}$ and $Q_{C}$ currently used in the simplified approach. The model has been improved by the addition of the Thomson contribution and the temperature dependence of all the parameters. Using a thermodynamical analysis, we have precise the different contribution to entropy and clarify the important role of the Joule effect on the performances of the thermoelectric element. The possible extraction of heat from a fraction of the thermoelectric leg gives promising result for better local $\mathrm{COP}$ or larger maximal $\Delta \mathrm{T}$ if connected with pulse mode current.

\section{Acknowledgments}

This study is financed by CNRS in the framework of " Programme Interdisciplinaire Energie $d u C N R S "$ 


\section{References}

[1] Seebeck, T. J., Ueber den magnetismus der galvenische kette, Abh, K. Akad. Wiss. Berlin, $289,1821$.

[2] Peltier, J. C. A., Nouvelles expériences sur la caloricité des courants électriques., Ann. Chem. Phys., 56, 371, 1834.

[3] Thomson, W., On a mechanical theory of thermo-electric currents, Philos. Mag. 5, 3, 529, 1852.

[4] Thomson, W., Account of researches in thermo-electricity, Philos. Mag. 5, 8, 62, 1854.

[5] Y Ioffe, A. F., Semiconductor Thermoelements and thermoelectric Cooling, Infosearch, London, 1957.

[6] Y.G. Gurevich, G.N. Logvinov. Theory of thermoelectric cooling in semiconductors structures. Revista mexicana de fisica, 53 (5) (2007) 337-349.

[7] Onsager, Reciprocal Relations in Irreversible Processes I\&II. Phys. Rev. 37, 405 - 426, Phys. Rev. 38, (1931) 2265 - 2279.

[8] H. B. Callen, The Application of Onsager's Reciprocal Relations to Thermoelectric, Thermomagnetic and Galvanomagnetic Effects, Phys Rev 73 (11) (1948) 1349-1358.

[9] C.A. Domenicali, Irreversible Thermodynamics of Thermoelectricity, Rev. Mod. Phys. 26 (1954) 237-275.

[10] C. B. Vining. The Thermoelectric Process. Materials Research Society Symposium Proceedings: Thermoelectric Materials - Mater. Res. Soc. 278 (1997) 3-13.

[11] L. D. Landau and E. M. Lifshitz, Electrodynamics of Continous Media, 2nd Edition, Butterworth Heinemann, Oxford, 1984.

[12] A. Chakraborty, K.C. Ng. Thermodynamic formulation of temperature-entropy diagram for the transient operation of a pulsed thermoelectric cooler. International Journal of Heat and Mass Transfer 49 (11-12) (2006), 1845-1850.

[13] B.J. Huang, C.L. Duang. System dynamic model and temperature control of a thermoelectric cooler. International Journal of Refrigeration 23 (2000) 197-207.

[14] A. Chakraborty, B.B. Saha, S. Koyama, K.C. Ng. Thermodynamic modelling of a solid state thermoelectric cooling device : Temperature-entropy analysis. International Journal of Heat and Mass Transfer 49 (19-20) (2006), 3547-3554.

[15] R. Yang, G. Chen, A.R. Kumar, G.J. Snyder, J.P. Fleurial. Transient cooling of thermoelectric coolers and its applications for microdevices. Energy Conversion and Management 46 (9-10) (2005) 1407-1421.

[16] G.J. Snyder, J.P. Fleurial, T. Caillat, R. Yang and G. Chen. Supercooling of Peltier cooler using a current pulse. Journal of Applied Physics 46 (3) (2002) 1564-1569. 
[17] M.J. Huang, R.H. Yen, A.B. Wang. The influence of the Thomson effect on the performance of a thermoelectric cooler. International Journal of Heat and Mass Transfer 48 (2005) 413-418.

[18] A.K. Pramanick, P.K. Das. Constructal design of a thermoelectric device. International Journal of Heat and Mass Transfer 49 (2006) 1420-1429.

[19] G. Min, D.M. Rowe. Cooling performance of integrated thermoelectric microcooler. Solidstate Electronics 43 (1999) 923-929.

[20]. R.Y. Nuwayhid, F. Moukalled, N. Noueihed. On entropy generation in thermoelectric devices. Energy Conversion \& Management 41 (2000), 891-914.

[21] S.B. Riffat and X. Ma. Improving the coefficient of performance of thermoelectric cooling systems : a review. International Journal of Energy Research 28 (2004) 753-768.

[22] L. Chen, J. Li, F. Sun, C. Wu. Performance optimization of a two-stage semiconductor thermoelectric-generator. Applied Energy 82 (2005) 300-312.

[23] R. Yang, G. Chen, A.R. Kumar, G.J. Snyder, J.P. Fleurial. Transient cooling of thermoelectric coolers and its applications for microdevices. Energy Conversion and Management, 46 (9-10) (2005) 1407-1421.

[24] X. C. Xuan, K. C. Ng, C. Yap, H. T. Chua. Optimization of two-stage thermoelectric coolers with two design configurations. Energy Conversion and Management, 43 (5) (2002) 2041-2052.

[25] A. Bejan. Advanced Engineering Thermodynamics. Third Edition, Wiley, John Wiley \& Sons Inc., 2006. 
Figure captions

Figure 1 : Configuration studied (two adiabatic thermoelectric elements) with $\alpha$ the Seebeck coefficient and $j$ the electrical current density

Figure 2 : Analogical scheme of the thermoelectric phenomena with the thermal capacitances $C_{i}$ and thermal resistances $\mathrm{R}_{\mathrm{i}}$

Figure 3 : Performance of the thermoelectric element as a function of the intensity for several models (analo for the analogical model, analy for the analytical model, simpl for the simple model, and Thomson when the Thomson effect is taken into account)

Figure 4 : COP and $\dot{Q}_{C}$ evolution for different intensities (from 1A up to 9A)

Figure 5 : Heat flux and temperature in the thermoelectric element: analogical model versus analytical model

Figure 6 : Variation of the different thermophysical parameters as functions of the temperature

Figure 7 : Influence of the different effects (Seebeck, Joule, Thomson) on the heat flux repartition

Figure 8 : Influence of the different effects (Seebeck, Joule, Thomson) on the temperature repartition

Figure 9 : Representation of the different effects in the Temperature Entropy diagram

Figure 10 : Representation of the T-S diagram in the N-element

Figure 11 : Evaluation of the performance (COP, Qc and Sgen) for conical geometries

Figure 12 : Influence of the Joule effect evacuation at the middle of the element

Figure 13 : Effect of the pulsed intensity on the temperatures as function of time

Figure 14 : Effect of the pulsed intensity on the temperatures as function of space 


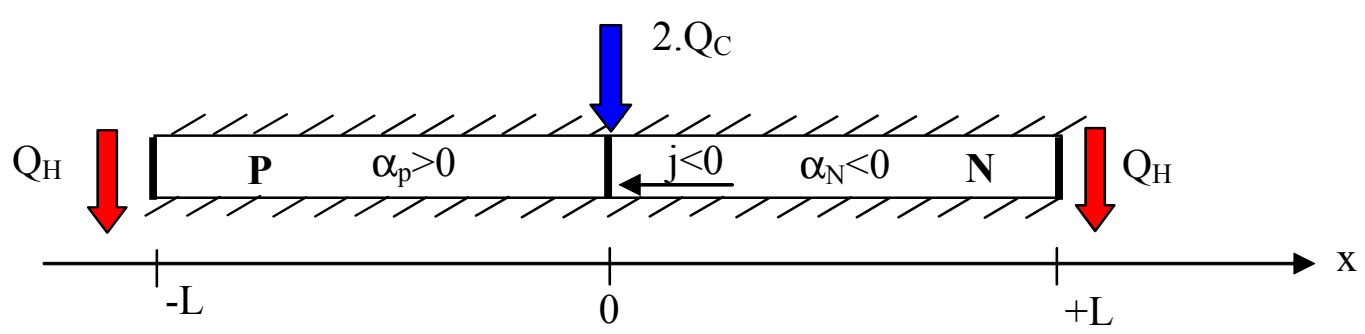

Figure 1 : Configuration studied (two adiabatic thermoelectric elements) with $\alpha$ the Seebeck coefficient, $j$ the electrical current density 


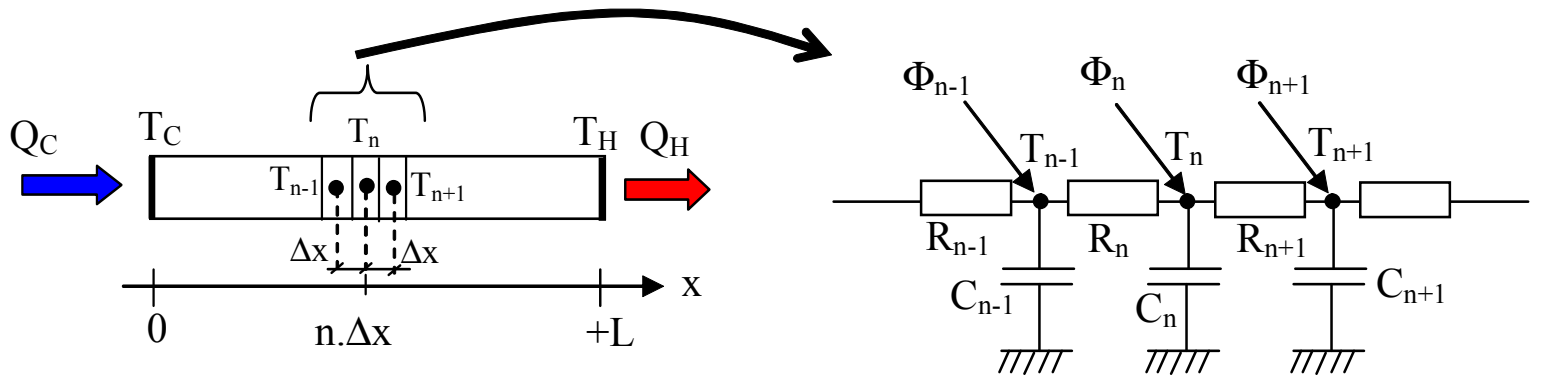

Figure 2: Analogical scheme of the thermoelectric phenomena with the thermal capacitances $C_{i}$ and thermal resistances $R_{i}$ 


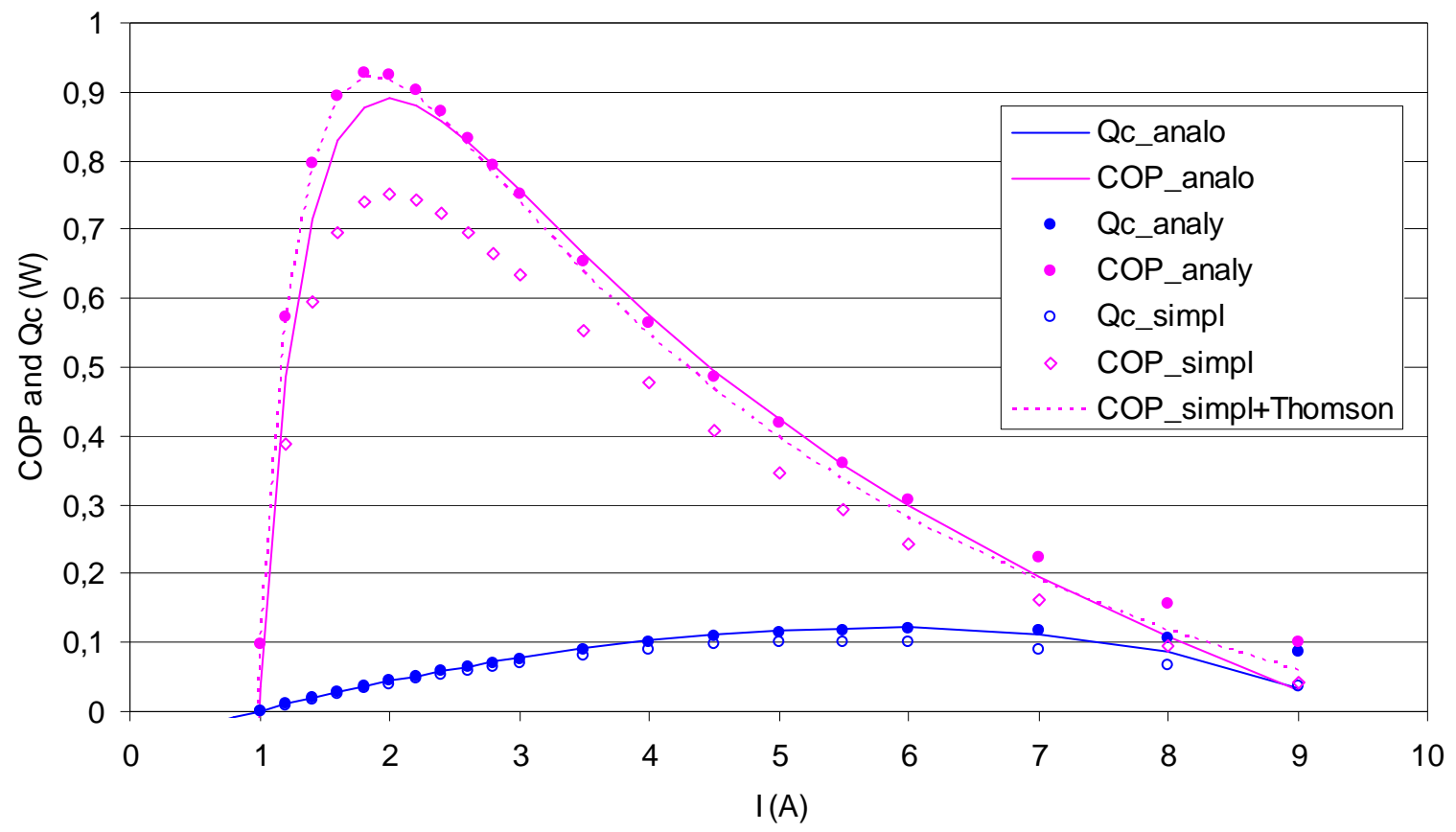

Figure 3 : Performance of the thermoelectric element as a function of the intensity for several models (analo for the analogical model, analy for the analytical model, simpl for the simple model, and Thomson when the Thomson effect is taken into account) 


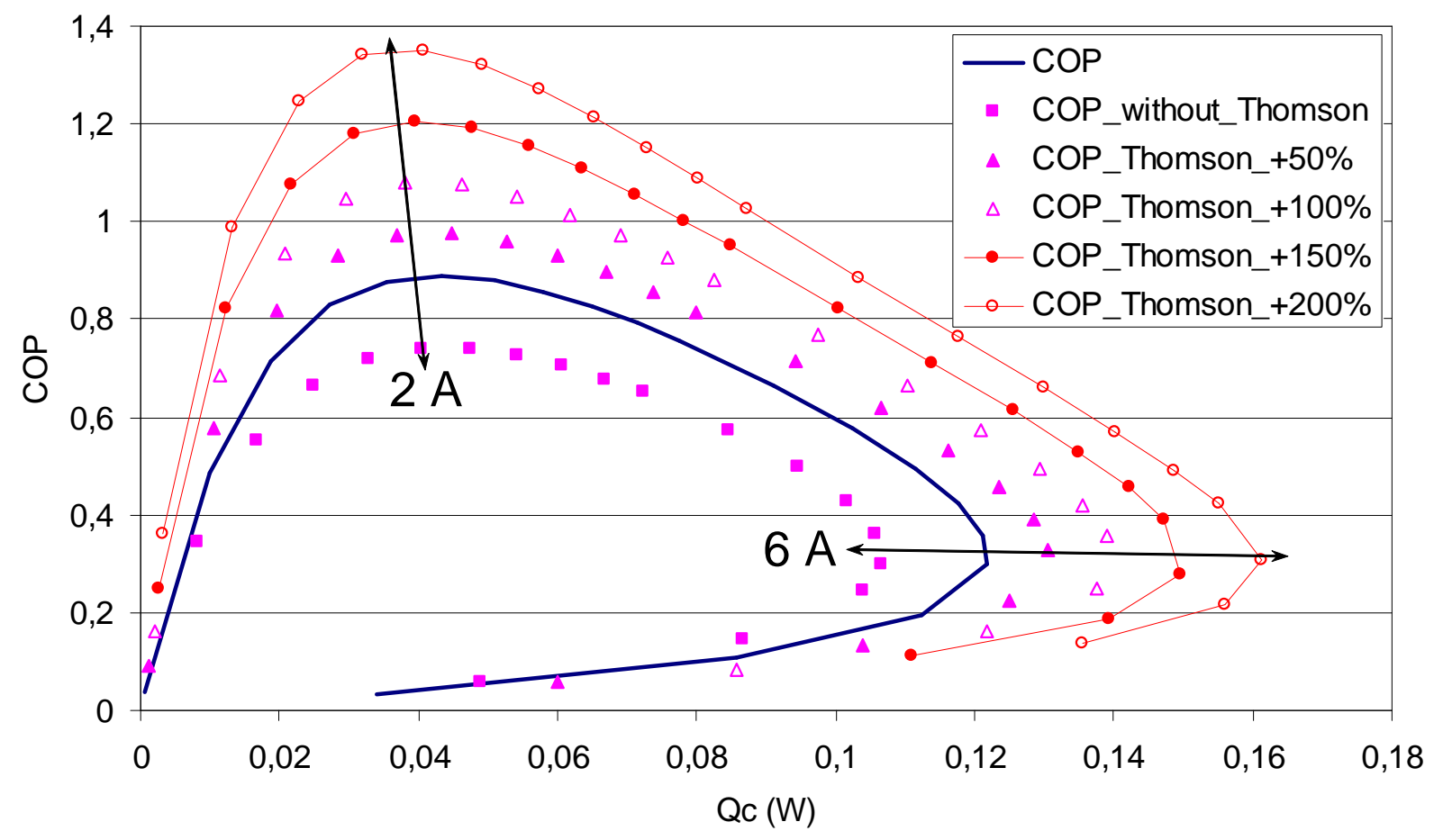

Figure 5 : Heat flux and temperature in the thermoelectric element:

analogical model versus analytical model 


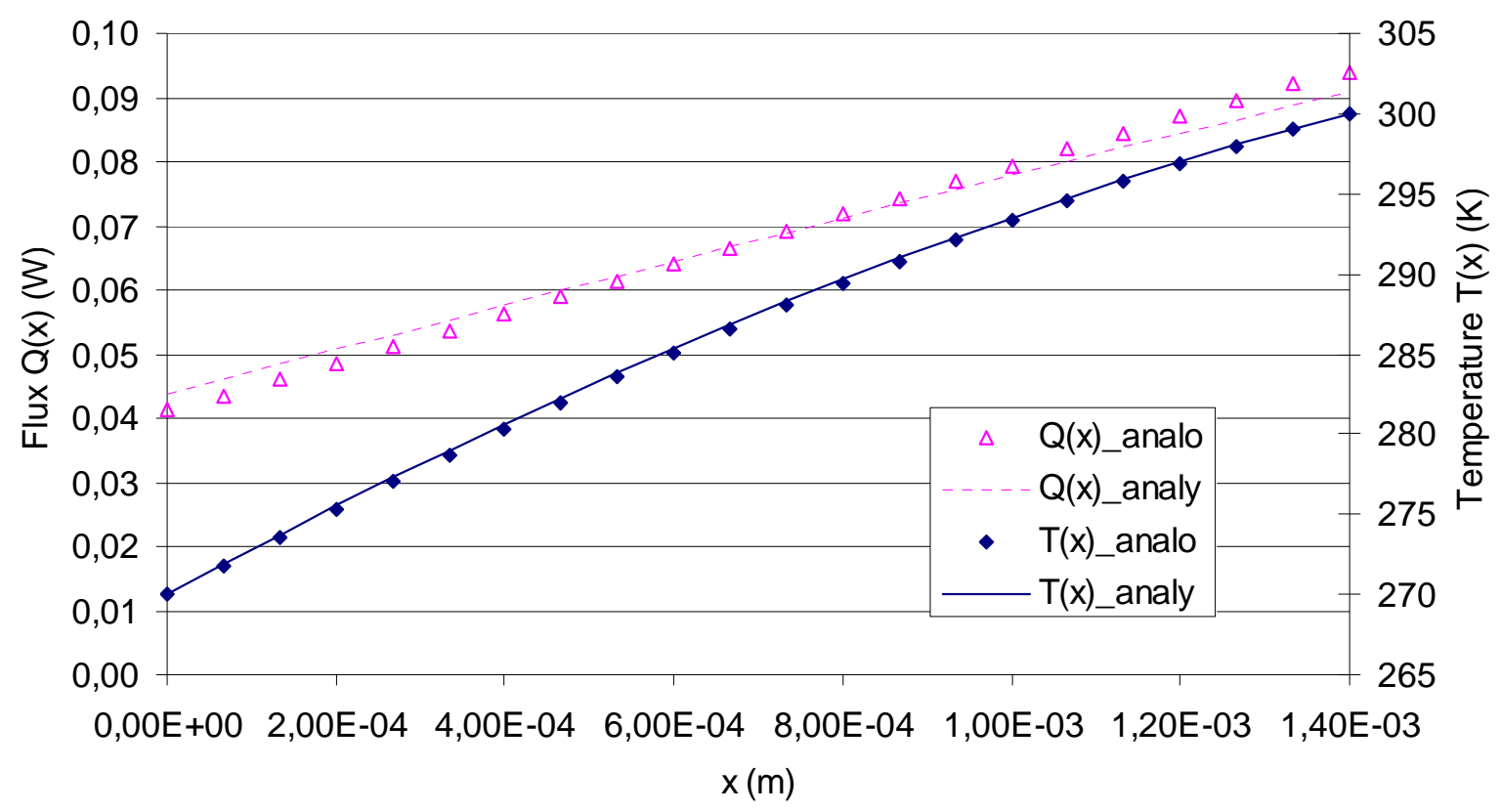

Figure 5 : Heat flux and temperature in the thermoelectric element:

analogical model versus analytical model 


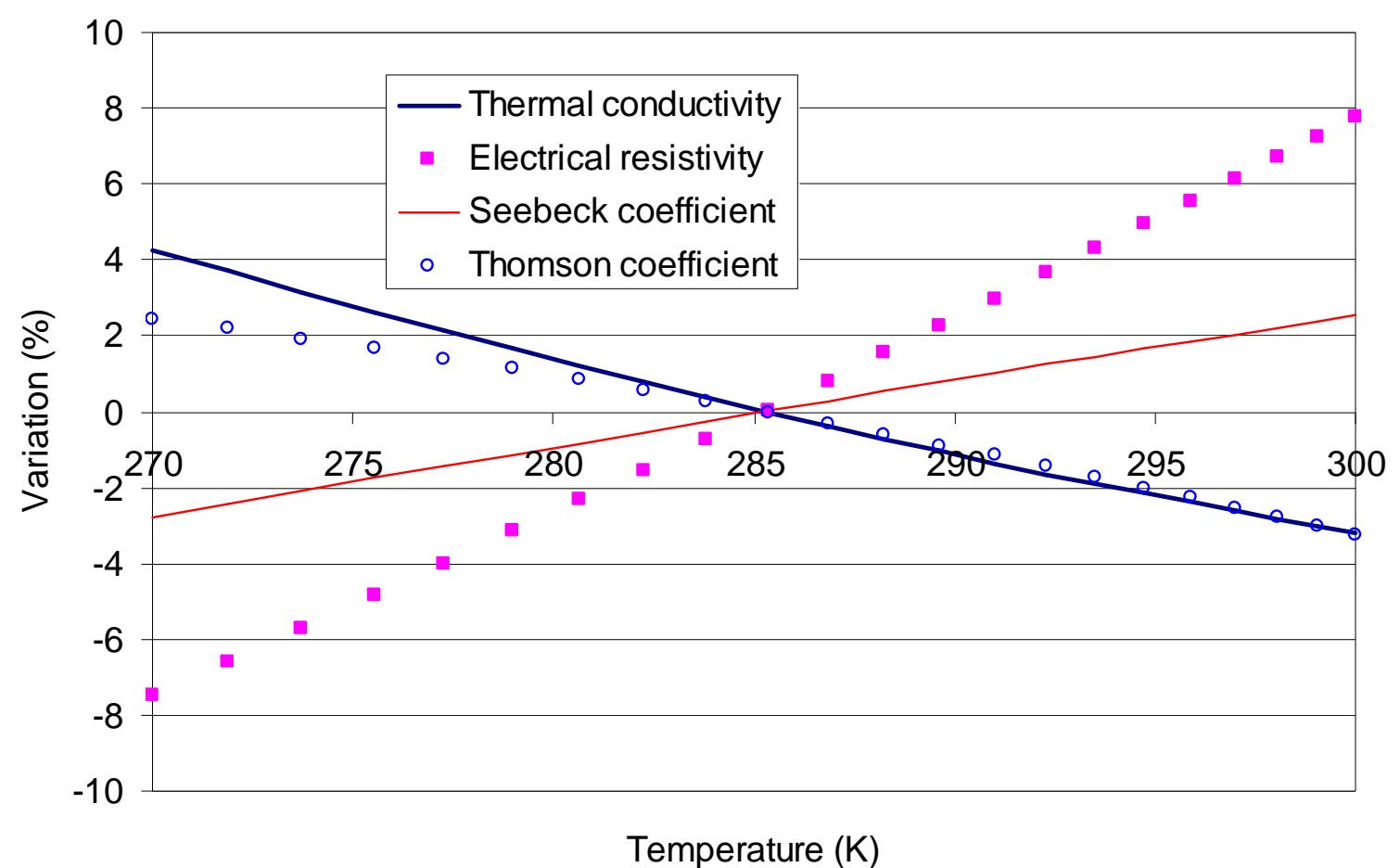

Figure 6 : Variation of the different thermophysical parameters as functions of the temperature 


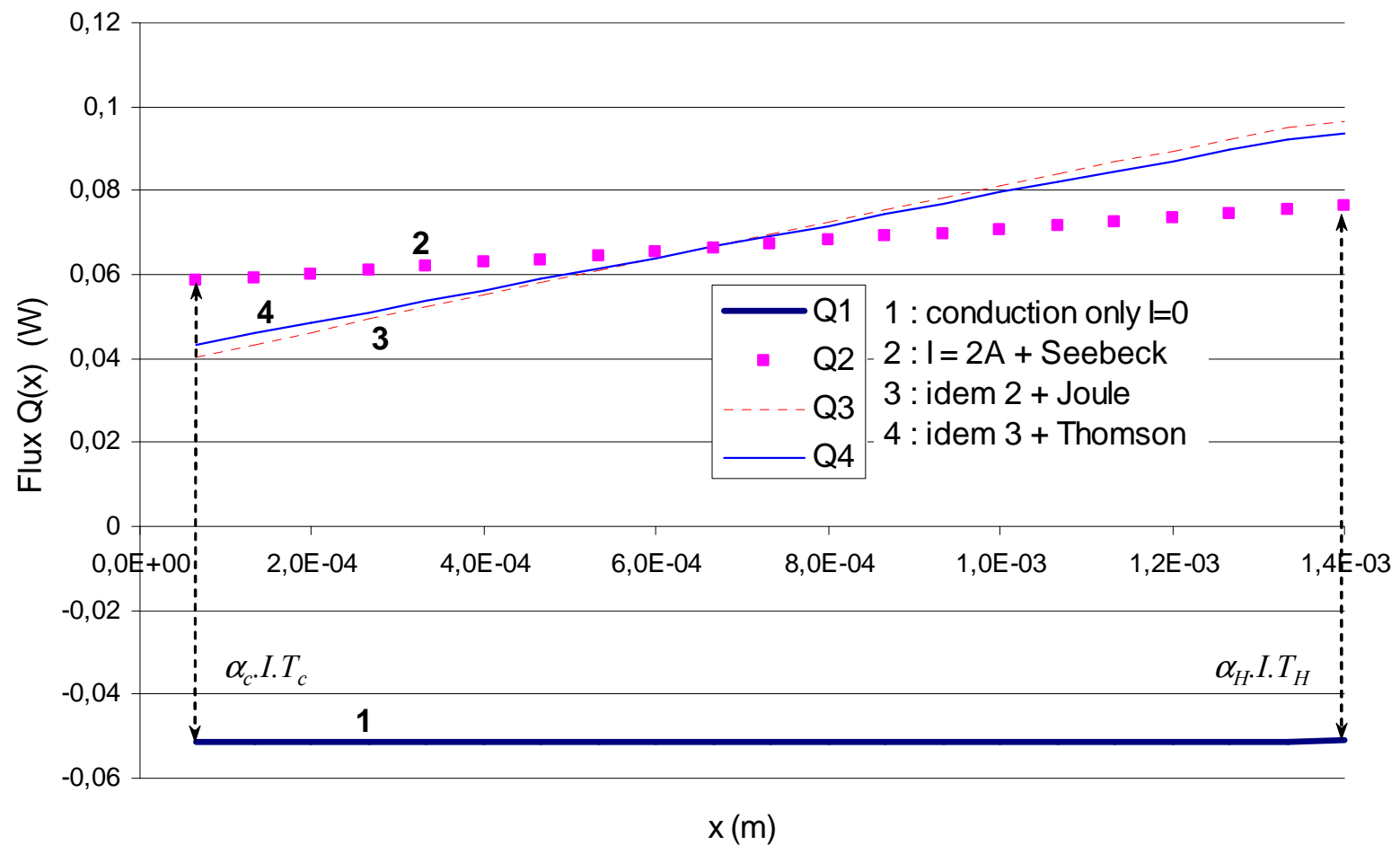

Figure 7 : Influence of the different effects (Seebeck, Joule, Thomson) on the heat flux repartition 


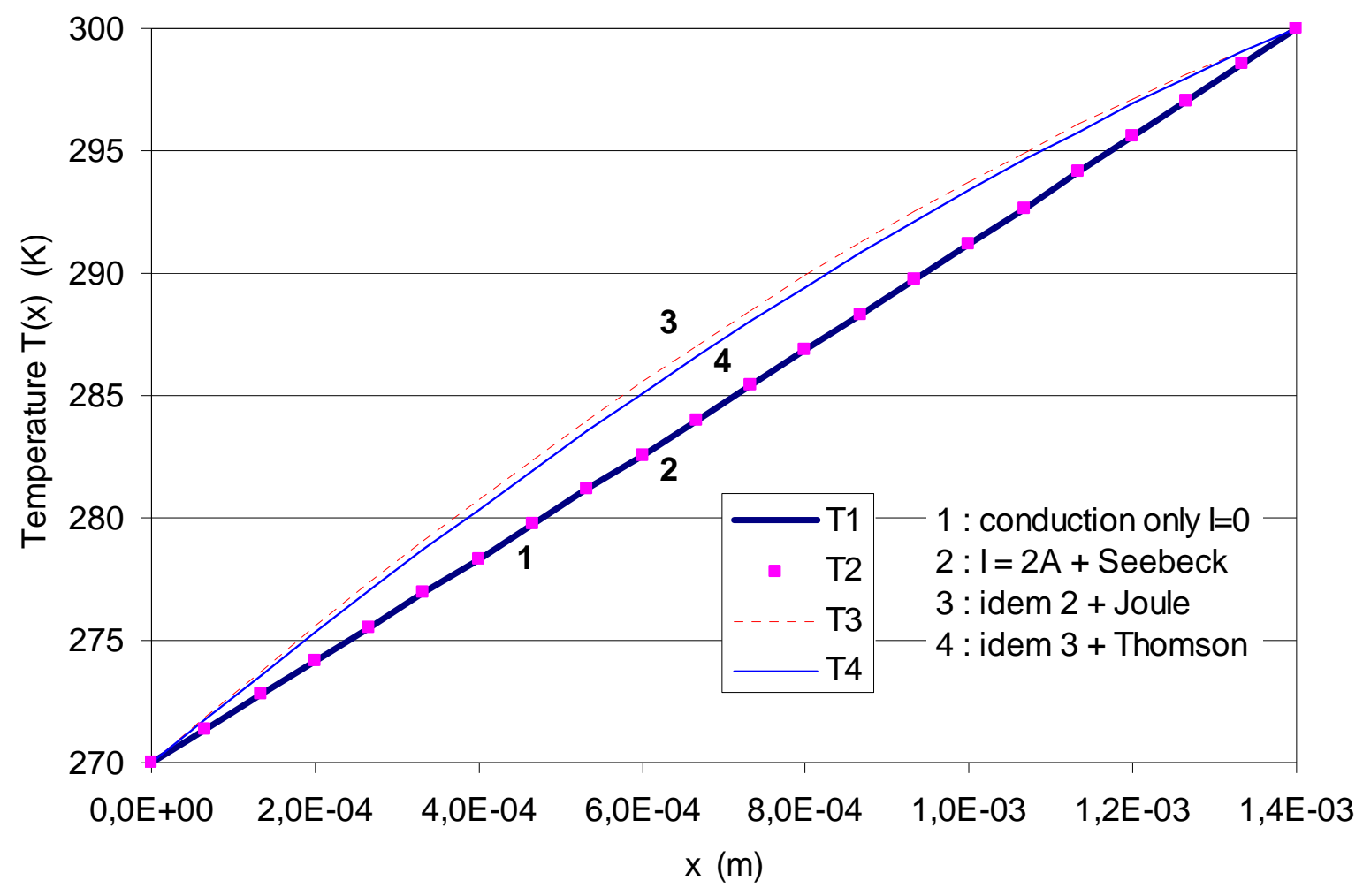

Figure 8: Influence of the different effects (Seebeck, Joule, Thomson) on the temperature repartition 


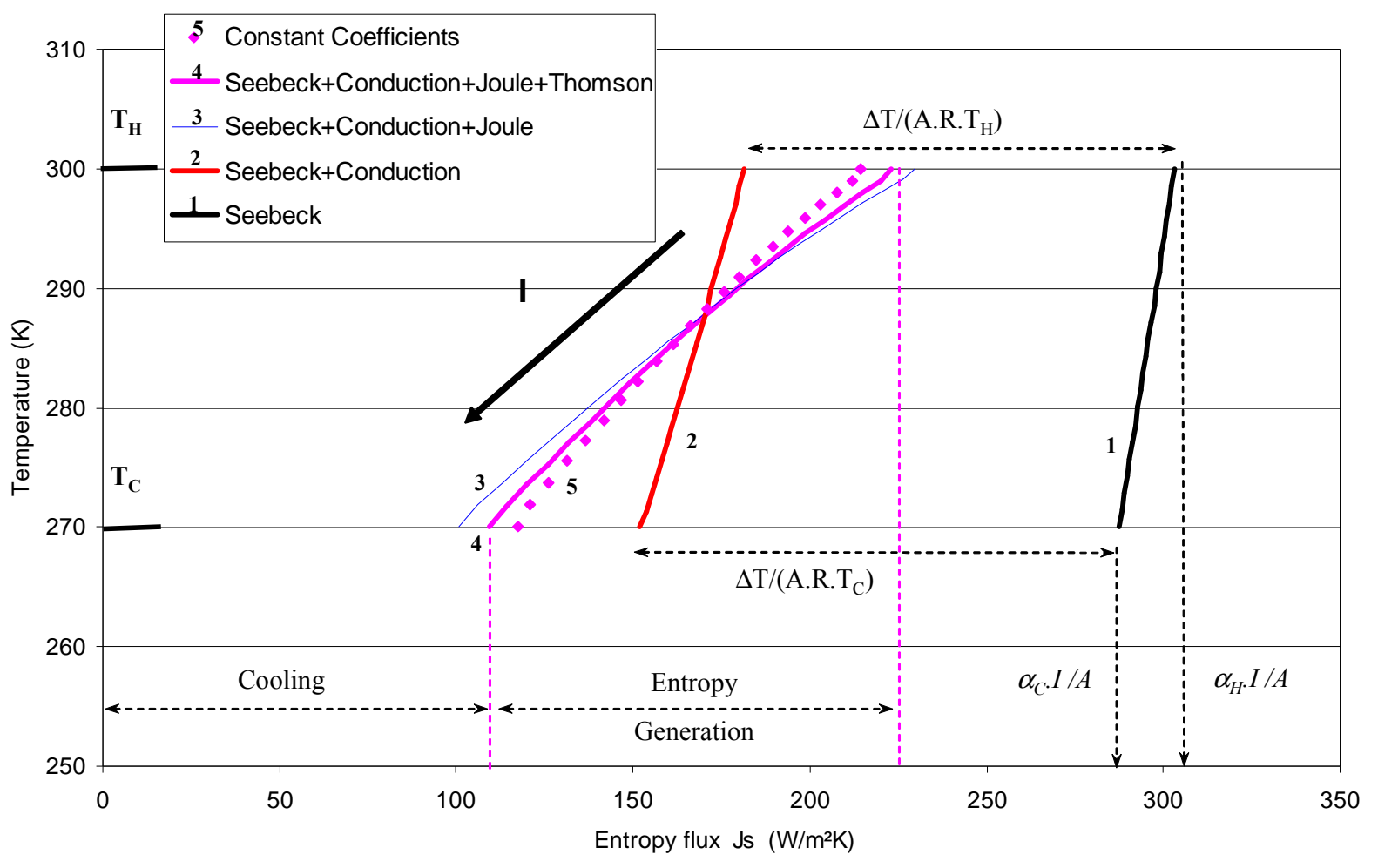

Figure 9 : Representation of the different effects in the Temperature Entropy diagram 


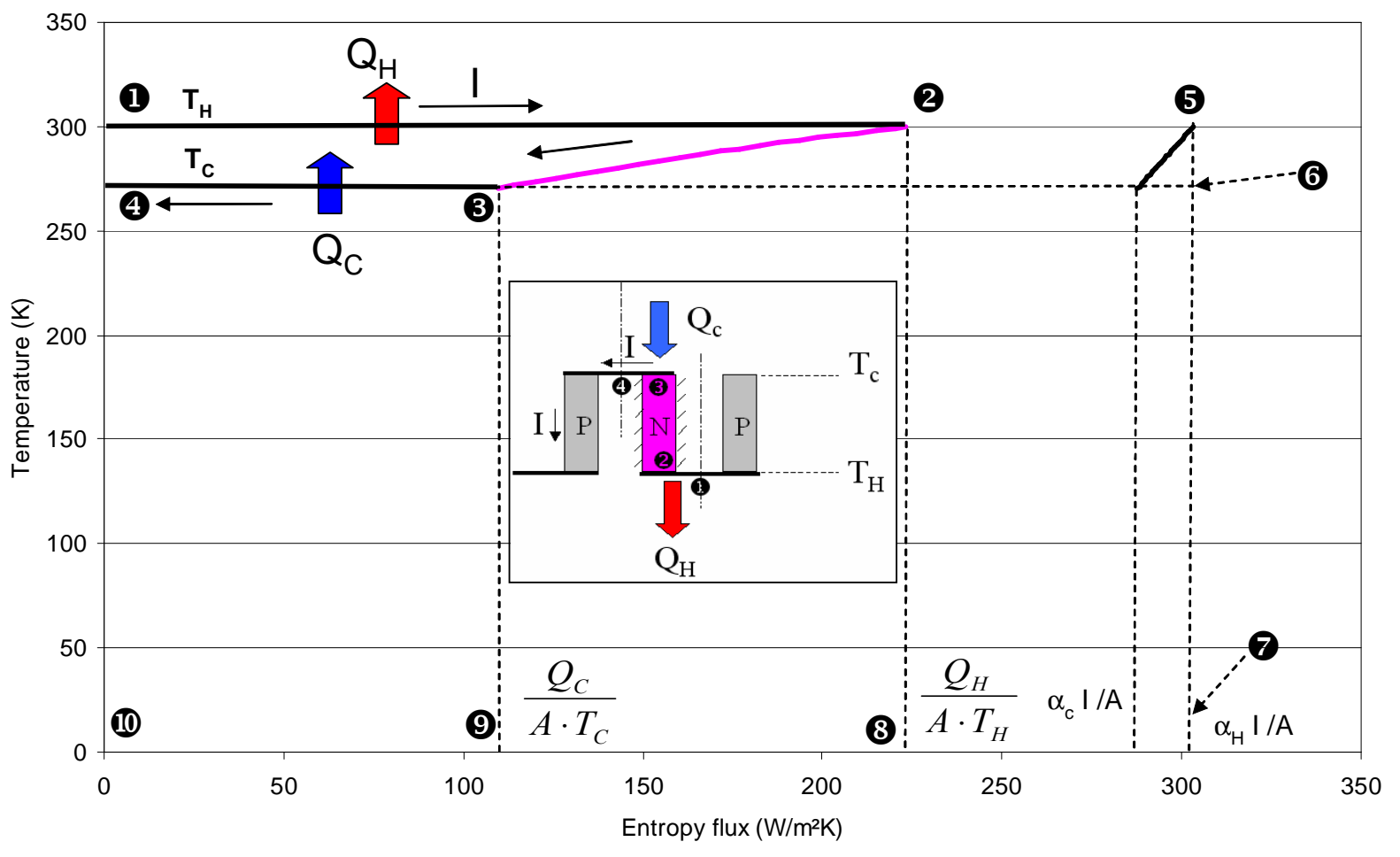

Figure 10 : Representation of the T-S diagram in the N-element 


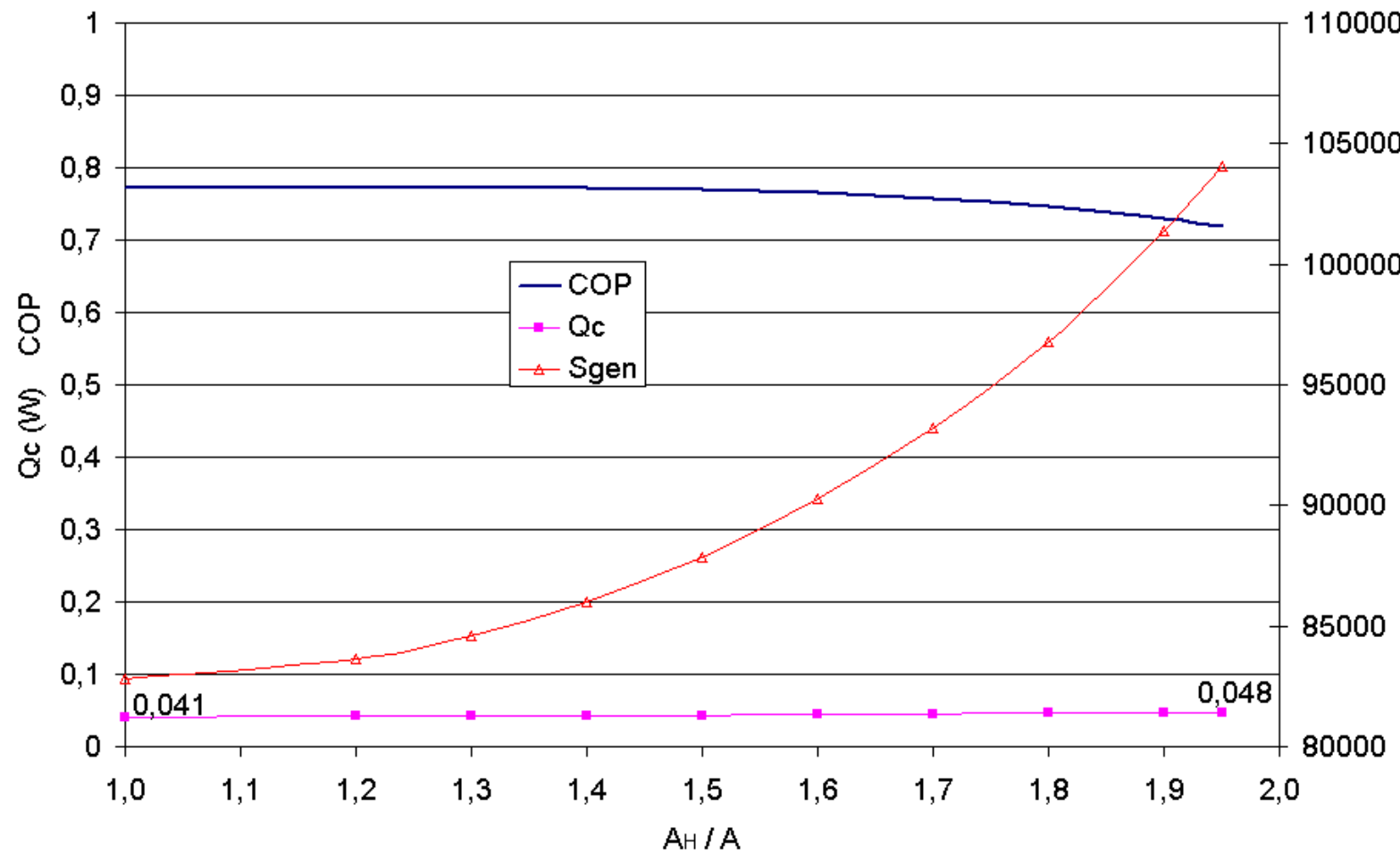

Figure 11 : Evaluation of the performance (COP, Qc and Sgen) for conical geometries $\left(\mathrm{A}_{\mathrm{H}}\right.$ is the section at the hot side, $\mathrm{A}=1.410^{-6} \mathrm{~m}^{2}$, the volume and the length of the leg are constant : 1.96 $10^{-9} \mathrm{~m}^{3}$ and $\mathrm{L}=1.410^{-3} \mathrm{~m}$ ) 


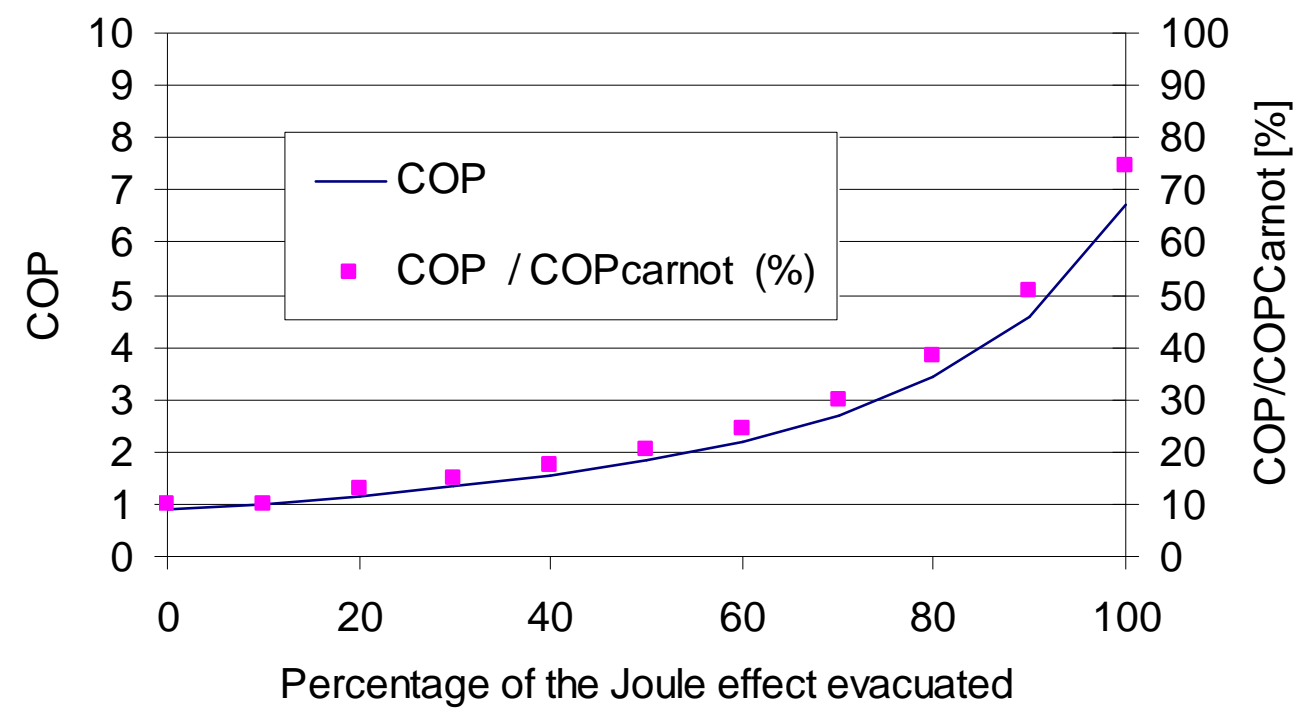

Figure 12 : Influence of the Joule effect evacuation at the middle of the element 


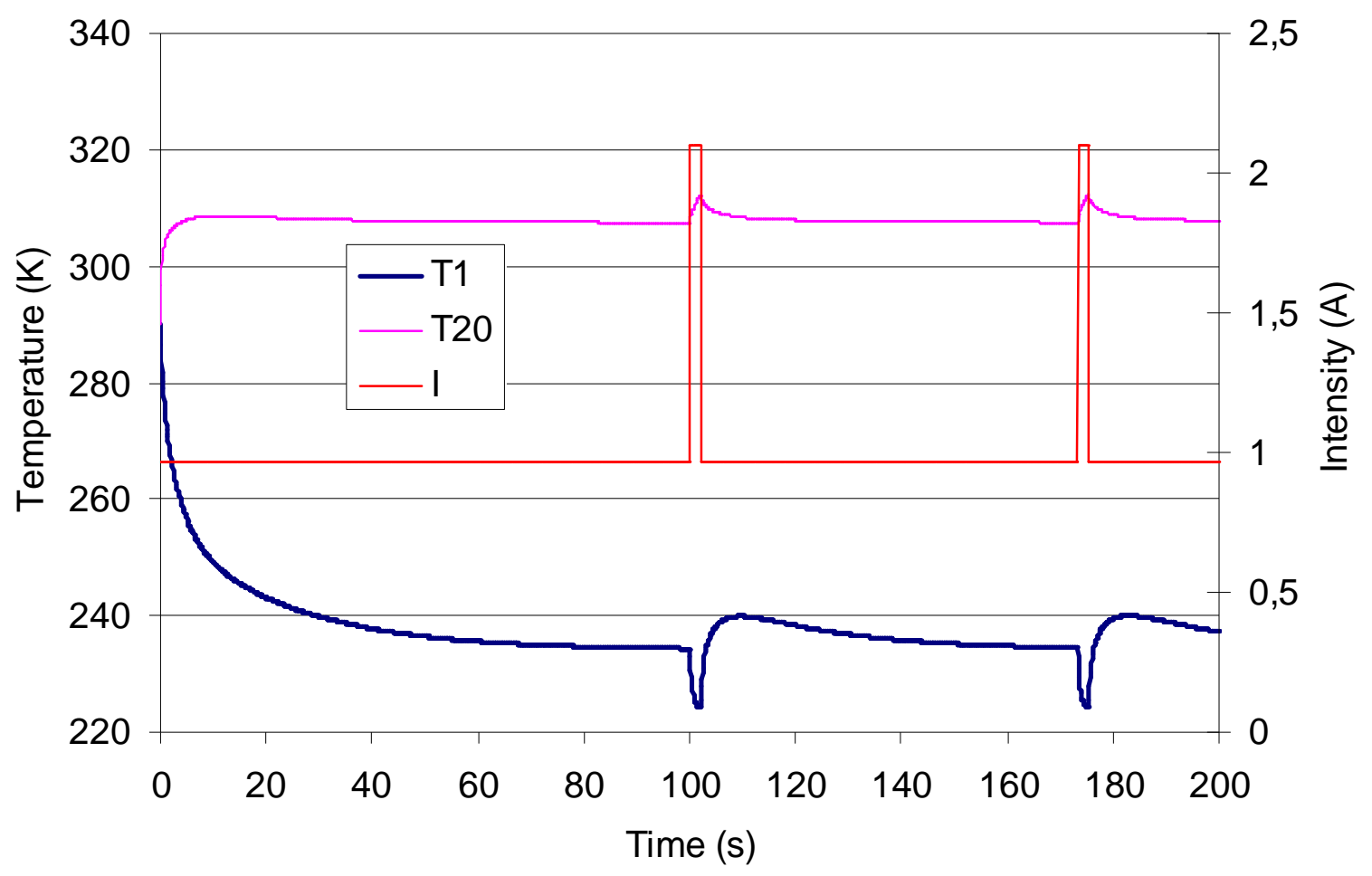

Figure 13 : Effect of the pulsed intensity on the temperatures as function of time 


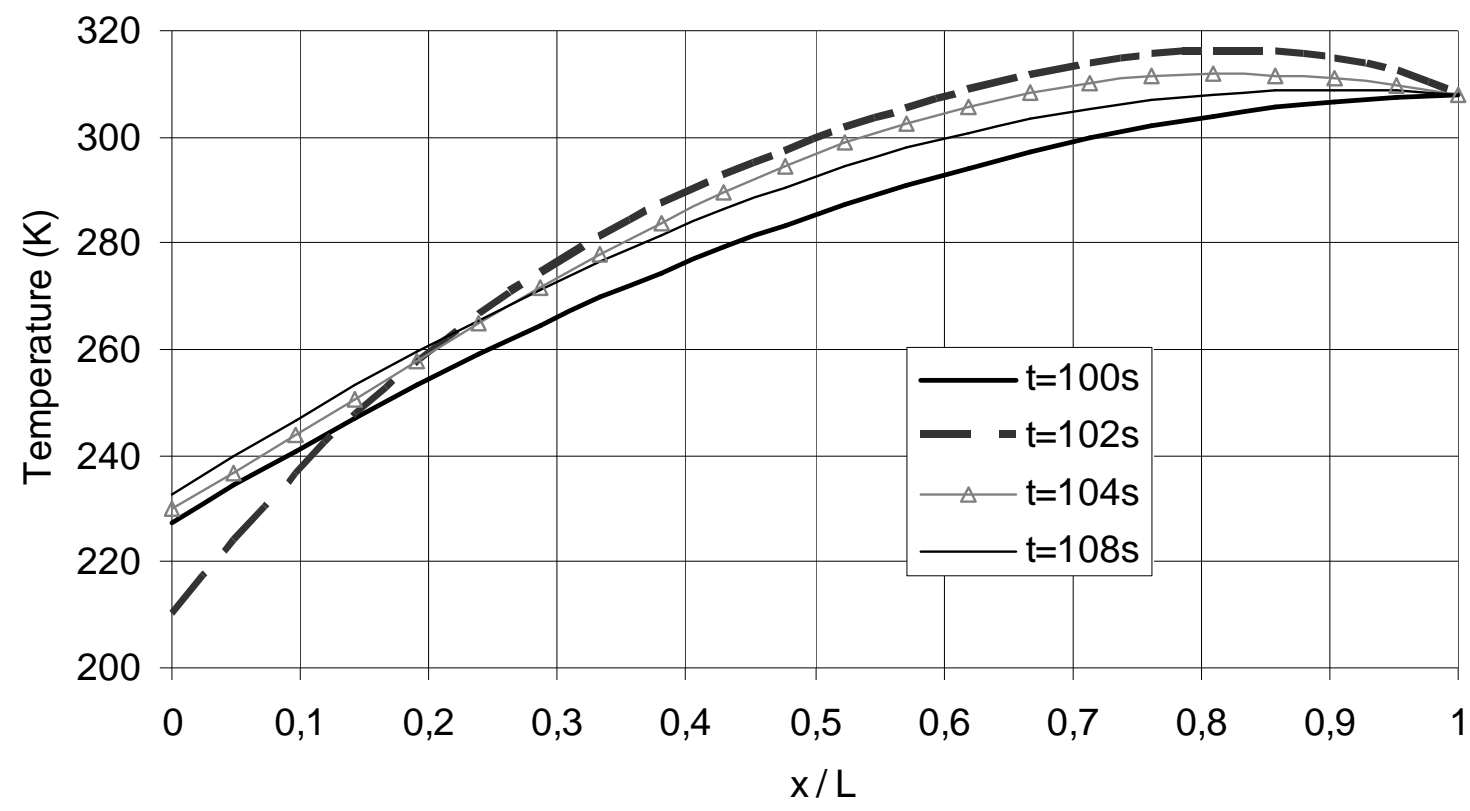

Figure 14 : Effect of the pulsed intensity on the temperatures as function of space 


\begin{tabular}{|l|l|c|}
\hline Temperature cold side $(x=0)$ & $T_{C}=270 K$ & \\
Temperature hot side $(x=L)$ & $T_{H}=300 \mathrm{~K}$ & \multicolumn{1}{|c|}{ Electrical Analogy: } \\
Length & $L=1.410^{-3} \mathrm{~m}$ & Parameters function of T \\
Constant section & $A=1.410^{-6} \mathrm{~m}^{2}$ & $\left(62605-277.7 T+0.4131 T^{2}\right) 10^{-4}$ \\
Thermal conductivity & $\lambda=1.701 \mathrm{Wm}^{-1} \mathrm{~K}^{-1}$ & $\left(5112+163.4 T+0.6279 T^{2}\right) 10^{-10}$ \\
Electrical resistivity & $\rho=1.02710^{-5} \Omega . \mathrm{m}$ & $\left(22224+930.6 T-0.9905 T^{2}\right) 10^{-9}$ \\
Seebeck coefficient & $\alpha=2.0710^{-4} \mathrm{~V} / \mathrm{K}$ & $\left(930.6-0.9905 T^{2}\right) 10^{-9} T$ \\
Thomson coefficient & $\tau=1.0410^{-4} \mathrm{~V} / \mathrm{K}$ & \\
\hline
\end{tabular}

Table 1 : Data of the thermoelectric element used for the simulations 